\title{
ALS INFORMATIONSSPEZIALISTIN IN DIE SELBSTSTÄNDIGKEIT? EIN BERICHT VON DER AIIP-KONFERENZ (PHILADELPHIA, 11.-14. APRIL 2019)
}

\section{von Birgit Bauer}

Zusammenfassung: Rund 100 selbständige Information Professionals und solche, die eine Selbstständigkeit planen, trafen sich im historischen Zentrum von Philadelphia zu drei Tagen Netzwerken und Lernen. Die Vorträge und interaktiven Workshops betrafen Themen wie Angebote schreiben und verhandeln, erfolgreiche Projektarbeit, die eigene Marke kommunizieren, wie am besten kooperieren - alle aus dem praktischen Erfahrungsschatz selbständiger Informationsexpertinnen und -experten -, sowie fachliche Top-Tipps-Lektionen.

Schlagwörter: InformationsexpertInnen, Information Professionals, BibliothekarInnen, Selbstständigkeit, Weiterbildung, Konferenzbericht

\section{AS AN INFORMATION EXPERT INTO SELF-EMPLOYMENT? A REPORT FROM THE AIIP CONFERENCE (PHILADELPHIA, APRIL 11-14, 2019)}

Abstract: Around 100 self-employed information professionals and some, who plan to get self-employed, met in Philadelphia's historic center for three days of networking and learning. The lectures and interactive workshops focused on topics such as writing and negotiating proposals, successful project work, communicating one's brand, best practices in cooperating - all from the business experience of independent information experts -, as well as technical top-tips sessions.

Keywords: information experts, information professionals, librarians, self-employement, vocational training, conference report

DOI: https://doi.org/10.31263/voebm.v73i1.3295

(C) Birgit Bauer

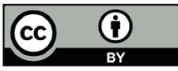

Dieses Werk ist - exkl. einzelner Logos und Abbildungen - lizenziert unter einer Creative-Commons-Lizenz Namensnennung 4.0 International 
Als langjähriges Mitglied der internationalen Organisation Association of Independent Information Professionals (AIIP) mit Sitz in den USA, hatte ich letzten April endlich die Gelegenheit, an der jährlichen AllP-Konferenz teilzunehmen ${ }^{1}$. Sie findet im April oder Mai in wechselnden Städten der USA statt, zuletzt in Philadelphia. Freitag und Samstag bildeten den Kern der Konferenz und waren vollgepackt mit Vorträgen und Workshops. Donnerstagabend gab es die ersten Übungen, den Empfang und das erste Dine Around-Abendessen, Sonntag früh ein kurzes „Wrap-up“ für alle, die noch nicht abgereist waren.

\section{Netzwerken, Netzwerken, Netzwerken}

Diese Konferenz setzt aufs Netzwerken und gegenseitigen Erfahrungsaustausch. Das ist, wie auch die Teilnehmerbefragungen zeigen, ihre besondere Stärke. Frühstück, Lunch und am Samstag das Gala-Dinner sind Teil des Programms. An den anderen Abenden wird das Dine Around angeboten und fleißig genutzt. Dafür sind in unterschiedlichen Restaurants Tische für die Teilnehmenden reserviert und man trägt sich dort ein, wo einem das Restaurant oder die anderen eingetragenen Tischgäste, falls bekannt, zusagen. Somit kann man die freien Abende, auch wenn man niemanden kennt, in interessanter und netter Gesellschaft verbringen und Kontakte vertiefen.

Seit den Anfängen der AlIP hat sich außerdem die Tradition der Vorstellungsrunde erhalten. Am ersten Tag der Konferenz (Freitagmorgen) stellten sich alle Teilnehmenden in jeweils einer Minute vor. Eine Liste mit Namen, Kontaktdetails und Raum für Notizen in den Konferenzunterlagen erleichtert nach etwa 100 Kurz-Vorstellungen die Zuordnung der Namen und später die direkte Kontaktaufnahme mit den für das eigene Business besonders interessanten Personen.

Auch die Bandbreite an Einsatzgebieten, Dienstleistungen und Erfahrungen lässt sich so gut erkennen und beeindruckt: die InformationsexpertInnen kamen aus einer Vielzahl von Bereichen - von Patentrecherche, Marketing Research, Competitive Intelligence, über Faktencheck (ScriptClearance) für Drehbücher, Film und Fernsehen, Autorlnnen (u.a. Mary Ellen Bates, Verfasserin des Buches Building \& Running a Successful Research Business), HerausgeberInnen (z.B. Marydee Ojala, Herausgeberin der Zeitschrift Online Searcher), Medical Writers, einem Detektiv (nach ersten Berufsjahren im Justizministerium), bis hin zu Informationsmanagement-Fachleuten, Consultants, Lehrenden, TrainerInnen, RednerInnen... Die Atmosphäre war entspannt und unterstützend, es gab im Wesentlichen keine direkten Konkurrenten. Die Mehrzahl der Teilnehmenden sind AlIP-Mitglieder oder solche, 
die es werden wollen, und kommen aus allen Ecken der USA. Ich war eine der wenigen aus Europa und die einzige aus dem deutschen Sprachraum.

\section{Tipps zur Projektarbeit}

Wie erstelle ich ein Dienstleistungsangebot? Wie gehe ich damit um, wenn ich den Aufwand noch nicht genau abschätzen kann? Was tun, wenn ein Projekt vom Plan abweicht? Was sind Best Practices beim Kooperieren oder beim Vergeben und Annehmen von Subaufträgen? Welche technischen Werkzeuge und welche Software können meinen Arbeitsalltag als Selbständige erleichtern? Diese und ähnliche Fragen stellen sich früher oder später fast allen selbständigen Informationsprofis. In Podiumsdiskussionen, Vorträgen und Workshops gaben Rednerinnen und Redner Tipps und Beispiele aus ihrer beruflichen Praxis weiter.

In einer angeregten Podiumsdiskussion teilten beispielsweise Linda Rink, Chrissy Geluk und Mat von Hendy, worauf sie bei der Angebotserstellung und dem Aushandeln von Kosten, Leistungen und Fristen achten. Dazu gehört: Kundinnen und Kunden vom eigenen Wert zu überzeugen, beispielsweise durch Arbeitsproben oder durch Beschreibung, was vergleichbare Aufträge gebracht haben. Das Angebot sollte alle wesentlichen Informationen enthalten, wie Fertigstellungstermin, Kosten, Methode, aber auch was bis wann von der Kundschaft zur Verfügung gestellt werden muss (z.B. Suchbegriffe, bestimmte Daten oder Datenbankzugang, Rückmeldungen zu Zwischen- und Abschlussberichten). So können etwaige Missverständnisse sofort geklärt werden oder das Angebot bei Annahme gleich als Vertrag dienen. Es sollte aber gleichzeitig nicht zu viel preisgegeben werden, damit nicht potenzielle Kunden und Kundinnen anhand der vorgeschlagenen Abwicklung billigere Studierende beauftragen könnten. Eine Frage aus dem Publikum betraf die typische Dauer zwischen Anfrage und Auftrag - eine Woche bis 3 Monate, in Ausnahmefällen länger. Eine weitere Frage war, ob die Vortragenden mit Projekten starten, ohne eine Form der schriftlichen Auftragserteilung - einhellige Antwort: nein.

Manchmal kann es vorkommen, dass sich die Anforderungen - am ehesten Umfang oder Fragestellung - im Laufe eines Projektes ändern. Auch dafür gab es Vorträge, Diskussionsrunden und hilfreiche Ideen. Das Wichtigste: Möglichst viele Details vorab klären und die Arbeit in mehrere Phasen teilen. Sobald die Aufgabe gravierend vom Plan abweicht, die Kundschaft informieren und sie daran erinnern, dass nach Projektstart zusätzliche Anforderungen zu einem neuen Angebot und zur angemessenen Entlohnung der Extra-Leistungen führen. 


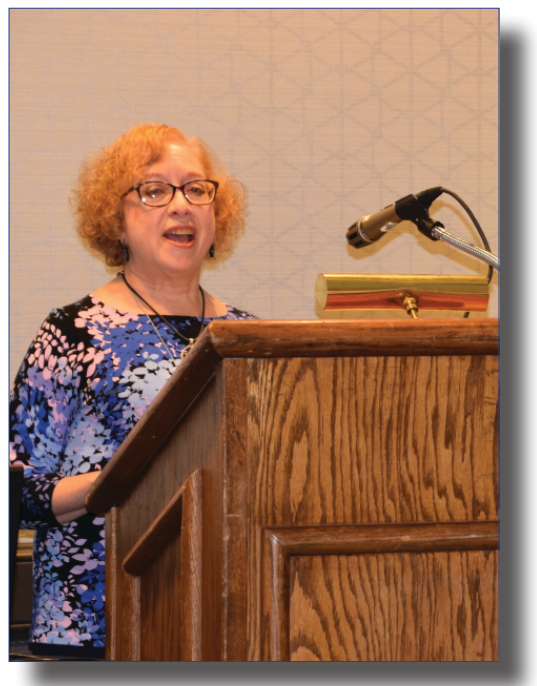

Abb. 1: Rhonda Kleiman (Rhonda Kleiman Group) empfahl in ihrem Beitrag über erfolgreiche Projektarbeit und zufriedene KundInnen: „It's all about establishing and maintaining expectations and communication." (Foto: Mark Goldstein)

Sehr humorvoll und lebendig brachten Karen Klein und Michelle Rawl in ihrer Doppelconference „Need Help? Phone a Friend“ das Thema Kooperation und Subaufträge auf den Punkt.

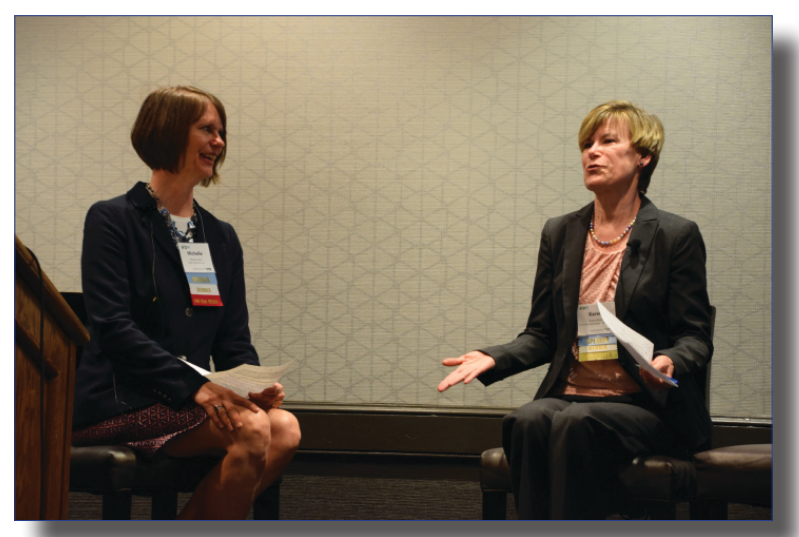

Abb. 2: Michelle Rawl (Rawl Research), Karen Klein (Fulcrum Information Resources): Wenn Projekte außerhalb ihrer Erfahrung oder Komfortzone liegen, arbeiten die beiden zusammen oder kooperieren mit anderen Informationsprofis - als Partnerln, Auftraggeberln oder Auftragnehmerln. (Foto: Mark Goldstein) 


\section{PR \& Marketing}

Die Frage, wie man sich neue Auftraggeberlnnen erschließt, ist für alle Selbständigen wichtig, auch für Information Professionals. Die Sichtbarkeit und Glaubhaftigkeit als Expertin und Experte können wir durch das Schreiben von Blogs mit Praxisbeispielen - beispielsweise Recherche-Tipps, oder was Fachleute außerhalb der typischen Google-Suche finden können - durch Vorträge, Veröffentlichungen, Netzwerken oder das Publizieren von Case Studies erhöhen. Sollte es an Aufträgen fehlen, die man veröffentlichen darf, könnten auch aussagekräftige Projekte für Freunde, Freundinnen oder Bekannte als Case Studies dienen.

Es gilt aber auch bestehende Kundinnen und Kunden zu begeistern. Ihnen genau zuzuhören, für ihre Probleme Lösungen anzubieten, ihre Erwartungen zu übertreffen, sie mit der eigenen Arbeit „gut aussehen zu lassen“ sind ein paar der Ratschläge, die mit Praxis-Beispielen untermauert wurden. Oder, wenn ein Auftraggeber eine für uns komplett neue Aufgabe vorschlägt, nicht sofort abzulehnen, sondern sich mit einem „Erzählen Sie mir mehr" die Zeit zu nehmen, den Auftrag zu analysieren und mögliche Methoden zu überlegen, und erst dann mit einem Ja oder Nein zu antworten.

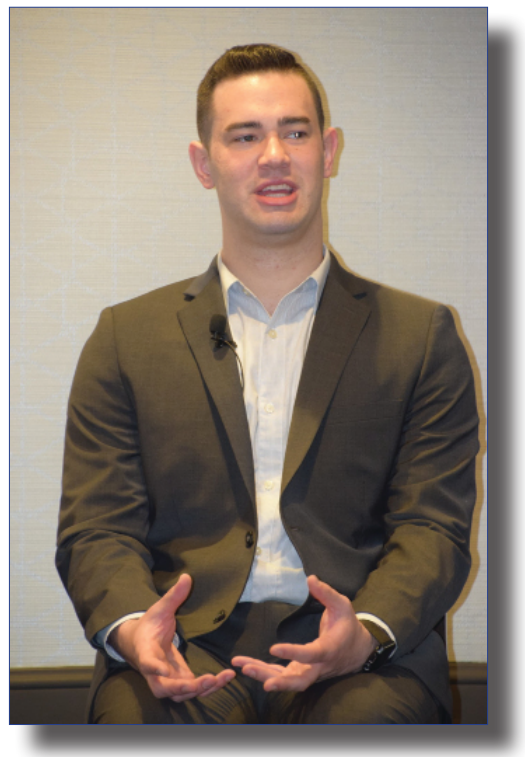

Abb. 3: Eddie Ajaeb (Nighthawk Strategies; Foto) und Connie Crosby (Crosby Consulting Group, nicht auf dem Bild) erzählten, wie sie neue Kundenbedürfnisse identifizieren, daraus neue Angebote entwickeln und dafür werben. (Foto: Mark Goldstein) 


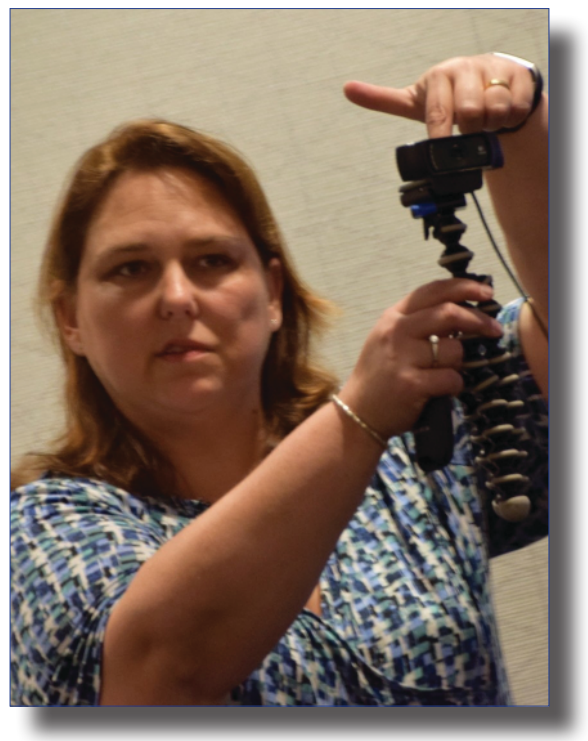

Abb. 4: Jennifer Burke (Intellicraft Research) stellte Technik-Tools vor. (Foto: Mark Goldstein)

Hilfreiche Technik-Tools stellte Jennifer Burke vor. Im Marketing waren es unter anderem: Evernote (zum Organisieren von Listen, Projekten, Ideen), Canva (Grafikdesign-Software, online zu nutzen, inklusive Fotos, Schriftarten, Grafiken), BuzzSumo (Popularität von Überschriften und Inhalten bestimmen), HemingwayApp (Software zum Korrekturlesen und zum Vereinfachen von Sätzen), Unsplash (Fotos zur kostenlosen Nutzung), SmarterQueue (Social Media Management).

Ein paar der Vorträge und Diskussionen sind schriftlich zusammengefasst im AllP-Blog zu finden. ${ }^{2}$ Ebenso die Keynote "Slow down to speed up" der Beraterin Liz Bywater, die allen Teilnehmenden sogar ein Exemplar ihres Buches Slow Down to Speed Up: Lead, Succeed, and Thrive in a 24/7 World schenkte.

\section{Persönliches Resümee}

Wer sich vor kurzem als Information Professional selbständig gemacht hat oder gerade dabei ist, dies zu tun, für die oder den ist die Konferenz aufjeden Fall ein Gewinn. Ebenso für bereits selbständige Informationsexpertinnen und -experten, die Teil des internationalen Netzwerks der AllP werden möchten. 
Die nächste Konferenz der AlIP findet vom 23. bis 26. April 2020 in Denver, Colorado, statt. ${ }^{3}$

\author{
Dipl.-Ing. in Birgit Bauer \\ Bauer Business Research e.U. \\ E-Mail: office@BauerBusinessResearch.com
}

1 Zum Programm der Konferenz 2019 siehe: https://www.aiip.org/conference/past-conferences/2019-conference

2 https://blog.aiip.org/tag/aiip19-wrap-up/

3 Zum Programm der Konferenz 2020 siehe: https://www.aiip.org/conference 